\title{
A report on International Conference "Modern Treatment Strategies and Horizons in Hematology and Oncology" (June 30-July 1, 2017)
}

Andrey Yu. Zaritskey ${ }^{1}$, Boris V. Afanasyev ${ }^{2}$, Dmitry V. Motorin ${ }^{1}$

${ }^{1}$ Federal Almazov North-West Medical Research Centre, St. Petersburg, Russia.

${ }^{2}$ R. Gorbacheva Memorial Research Institute of Children Oncology, Hematology and Transplantology, The First St. Petersburg State I. Pavlov Medical University, St. Petersburg, Russia

Professor Andrew Yu. Zaritskey, Federal Almazov North-West Medical Research Centre, St. Petersburg, Russia

\section{Summary}

A joint conference of clinicians from M.D. Anderson Medical Center (Houston, Texas, USA) and specialists from leading Russian hematological centers took place in St. Petersburg on June 30-July 1, 2017. A series of special sessions was dedicated to current issues of diagnostics and pathogenetic molecular classification in hemato-oncology, including chronic lymphocytic leukemia (CLL), acute leukemias (AL), aplastic anemias (AA), myelodysplastic syndrome (MDS), malignant lymphomas. Standard treatment schedules, as well as clinical effects of newly developed targeted drugs and combined therapeutic approaches were analyzed, with a focus on
Phone: +7 (812) 7026828

Fax: +7(812) 7023765

E-mail: zaritskey@gmail.com
Hodgkin's disease, chronic lymphocytic and myeloid leukemias and multiple myeloma. Recent developments in AL treatment, especially, in acute myeloblastic leukemia, were also presented. Current position of hematopoietic stem cell transplantation as a curative treatment in some leukemia/lymphoma settings, opportunities for tumor immunotherapy (e.g., CAR-T cells) and appropriate technologies were also discussed.

\section{Keywords}

USA-Russian conference, leukemia, lymphoma, pathogenesis, classification, treatment, targeted drugs, allogeneic hematopoietic stem cell transplantation.

\section{Introduction}

A number of well-known specialists in oncohematology from MD Anderson Cancer Center Forum (Houston, Texas) have attended St. Petersburg for a joint conference with Russian hematooncologists from St. Petersburg, Moscow and many Russian regions. This conference proceeded at the Azimuth Hotel and was quite useful for practical doctors who got acquaintance with some new treatment approaches to chemotherapy, hematopoietic stem cell transplantation and immunotherapy in leukemias and lymphomas.

\section{Lymphomas and chronic lymphocytic leukemia}

After opening words from Organising Committee, Prof. Irina V. Poddubnaya has presented a review Significance of minimal residual disease in follicular lymphoma and CLL. She presented some epidemiological data about growing incidence of lymphomas and chronic lymphocytic leukemia (CLL) based on Russian and international data. Over last time, application of new drugs caused increased overall survival (OS) in follicular lymphoma (FL) and multiple myeloma (MM). New prognostic markers, e.g., ALK expression allowed distinguish more or less favorable cases of certain 
lymphomas. Main attention was paid to minimal residual disease (MRD) and its prognostic significance in FL and CLL. In CLL, adequate MRD predictors may be quantitatively assessed by means of flow cytometry (CD5, CD19, CD20, CD79b), as well as IGHV mutation detectable by PCR technique. These markers are taken as standards. Clear correlations are shown between MRD absence and OS as well as progression-free survival (PFS). Proper timing of MRD testing, as well as number of preceding therapy cycles are important. BEN001NORMA study data and other trials are presented, showing efficiency of MRD testing. The issues of CLL and lymphoma treatment are also discussed, especially with new drugs (Brentuximab, Ibrutinib etc.). Combined treatment options may also increase response rates. Recent data show superior effects of obinutuzumab over rituximab in combination with chemotherapyu of FL. In CLL, a combined treatment with bendamustine and Venetoclax is also effective. Optional allo-HSCT may be considered in CLL progression.

Doctors M. Keating and J. Burger (Houston, USA) have presented quite comprehensive lectures about current views on CLL diagnostics, including some recently discovered molecular predictive markers determining prognosis of CLL therapy, as well as novel schedules for of CLL treatment. Special attention was paid to modern drugs, both immune checkpoint inhibitors, apoptosis inhibitors, monoclonal; antibodies, and specific inhibitors of signaling pathways in tumor cells.

A lecture by Dr. Pei Lin The 2016 WHO classification of lymphoma, an update concerned molecular and phenotypic markers of different lymphomas. The lecturer presented a subgroup of "double-hit" malignancies with MIC rearrangements and BCL2 translocations as a sufficient pathogenetic factor in high-grade B cell lymphomas, thus presenting a molecular basis for improvement of current lymphoma classifications, along with other molecular markers adopted for stratifying lymphomas, especially their aggressive clinical forms.

Dr. Peter Johnson, in his lecture Progress in Hodgkin lymphoma at ICML 2017, has summarized the latest progress in Hodgkin's lymphoma (HL) prognostic markers (baseline PET, cell-free DNA) and treatment, new therapies, and new biology (reprogramming the R-S cells). Early-stage disease is treated by ABVD protocol controlled by PET, followed by repeated ABVD + involved-field radiotherapy. In current studies, Rituximab is used upfront, or (in cases of PET-negative status), before radiotherapy. Total Metabolic Tumor Volume (TMTV) is compared to EORTC classification as interim quantitative PET prognostic parameters; appropriate trials are underway. Total Lesion Glycolysis (TLG) indexes are also used in UK trials, showing high efficiency. MTV \& TLG 2.5 have been associated with increased 3 yr HL events and inferior PFS. Response adapted therapy using FDG-PET provides an opportunity to personalize the approach to therapy, to achieve a best balance between efficacy and toxicity. Results of a phase II study of Brentuximab vedotin (BV) using a response adapted design for $1^{\text {st }}$ line treatment of HL showed dose-dependence of adverse effects (neuropathy, haematological etc.). In summary, BV has good single agent response rates. However, it is not a sufficient treatment in advanced disease. Consolidation with high dose therapy is effective. Meanwhile, Nivolumab demonstrated frequent and durable responses, irrespective of depth of response, BV treatment history, and refractoriness to prior therapies. Combination of BV with nivolumab appears safe and demonstrated a high objective response rate. Restoration of B cell programs may offer new types of therapy. Patient's age ( $>50$ years) remains a major risk factor. A gene expression-based model combined with FDG-PET imaging to predict treatment response in advanced HL was tested in the RATHL study. Changes in tumor-specific cell-free DNA (cfDNA) could complement iPET in HL treatment prognosis.

Dr. Franco Cavalli (Bellinzona, Switzerland) has summarized his views on current treatment and classification of indolent lymphomas discussed at the International Conference on Malignant Lymphomas (ICML, Lugano, 2016). Despite sufficient success in involved-field radiotherapy (IFRT), a risk of relapses is still high, with common adverse factors at diagnosis, bulky disease etc. Favorable results of CVP treatment (6 cycles) versus R-CVP after IFRT for low-grade follicular lymphoma (TROG study) are shown. Watchful waiting may be a rational strategy in some cases. Rituximab maintenance therapy after R-CHOP, or R-FCM proved to be an effective live-prolonging treatment (progression-free survival) for, at least, 3 years (PRIMA trial, 2011). In PET-controlled studies (PET state after induction), the PFS values were $65 \%$ at 4 years as compared to $24 \%$ in PET(+) cases. Several new inhibitory drugs (Idelalisib, Ibrutinib, Copalizib) have shown good immediate clinical response (57-72\%) in indolent lymphomas. A concept of "no chemotherapy» strategies in treating follicular lymphomas was developed in SAKK-Nordic 35/10 Trial with Lenalidomide + Rituximab vs. Rituximab alone, or "RELEVANCE" Trial (Rituximab + Lenalidomide vs any chemotherapy) Lenalidomide + Rituximab Vs. R- CHOP or CVP or R-Bendamustine. A special attention is given to Marginal Zone B-Cell Lymphomas (MZL, according to WHO classification). E.g., staging of MALT Lymphoma should be based on pooled PET/CT detection approach. Lymphomas with $\mathrm{t}(11 ; 18)$ and those with lymph node involvement are unlikely to regress after H.pylori eradication thus composing a special risk group. Rituximab-Chlorambucil protocols are effective in MZL. Strategy for Waldenstrom macroglobulinemia (WM) is also discussed. WM chemotherapy could be initiated in cases of clinical symptoms, considering Rituximab, or chlorambucil, or, currently, with Ibrutinib.

\section{Acute myeloid leukemia}

A special session was dedicated to treatment of refractory/ resistant acute myeloid leukemia. Professor Hakop Kantarjian (Houston, USA) in his lecture How I treat AML. has shared his unique long-term experience in AML treatment, beginning with standard chemotherapy protocols, and followed with combination therapy supported by some novel targeted drugs aimed to inhibit distinct kinases and control points of leukemia cells. Specific traits of AML treatment in elderly patients was shown in a report by M. Ohanian Standard chemotherapy in young and elderly AML. Distinct pathogenetic features of acute myeloid leukemia in aging organism cause big problems with its treatment efficiency. Moreover, 
dose intensity of chemotherapy in this age group is also limited, due to different comorbidities, thus decreasing survival rates.

A report by Dr. Sergey Konoplev Detection of minimal residual disease in AML and ALL by flow cytometry immunophenotypic studies was devoted to practical laboratory aspects of MRD diagnostics in acute leukemias. High importance should be given to optimal workflow and reproducibility of immunophenotyping and immunohistochemical studies. The results of flow cytometry and molecular biology assays of MRD detection should be compared for each individual clinical situation. Some newly proposed marker mutations seem to be of pathogenetic significance could be valuable not only as predictive markers, but for MRD detection as well.

Professor Valery G. Savchenko (Moscow, Russia) presented an extensive report 25 years of AML randomized trials in Russia, summarizing data on consequent multicentric clinical AML trials are performed over last decades which showed sufficient increase in survival for the cohorts at younger ages, without sufficient success for older patients ( $>65$ years), independent on chemotherapy dose intensity. Five Russian trials on AML therapy were performed between 1992 and 2014 ( $n=1427$ cases). In recent AML study, mean age of patients was 59 years. Upon induction treatment based on $7+3$ regimen plus maintenance therapy, the main factors were determined, i.e., age, cytogenetics, WBC counts, LDH activity. Pregnancy is a sufficient risk factor in AML treatment, with respect to OS and PFS.

Meanwhile, AML is a major indication for allogeneic HSCT. Posttransplant complications could be prevented by special treatment, e.g., acute GVHD could be effectively prevented by posttransplant cyclophosphamide. To prevent possible graft failure, mesenchymal stem cells could be injected intraosseally, thus creating local microenvironment.

Novel targeted agents could present future options for AML treatment, including specific antibodies, FLT3-inhibitors, IDH-inhibitors, BCL2-inhibitors, hypomethylating drugs and immunotherapeutic tools (CAR T-cells, immune checkpoint inhibitors). Cytogenetic risk factors (with favorable, intermediate and adverse prognosis) should be taken into account when planning specific induction and consolidation therapy. European LeukemiaNet recommendations should be taken into account when considering intensive chemotherapy. Maintenance therapy proved to increase RFS in elder patients with unfavorable karyotype.

\section{Immunotherapy}

Dr. A. Bedikian (Houston, USA) has presented some modern biological concepts that the immune system can eliminate cancer cells in vivo. Modern imunotherapeutic strategies include: cytokines, (interferon, interleukin-2); cancer vaccines, oncolytic viruses, (T-vec), adoptive transfer of ex vivo activated $\mathrm{T}$ and $\mathrm{NK}$ cells, antibodies or other proteins that co-stimulate cells or block the immune checkpoint pathways. Adoptive T cell therapy, especially with CART-cells is studied for treatment of solid and, currently, hematologic cancers. The field of cancer immunotherapy has recently got a significant recognition, due to approval of immune check point inhibitors (ipilimumab, nivolumab, pembrolizumab) for treatment of melanoma by 2014. Ipilimumab (anti-CTLA-4) showed a significant efficiency in melanoma, however, with some autoimmune adverse effects. PD-1 inhibitors (Nivolumab, Pembrolizumab) proved to be effective in some solid tumors and Hodgkin's disease. Targeting immune checkpoints with monoclonal antibodies also showed some improvement in overall survival, either as single-agent therapy or combined treatment.

A keynote lecture Immune therapy approaches in AML/MDS was presented by Doctor Naval Daver spoke about special biological and clinical characteristics of AML/MDS, especially, in aged subjects. To increase efficiency of treatment in this cohort, one may discuss both conventional chemotherapy and its potential combinations with newly introduced targeted agents. Individual prognosis in these cases may be based on molecular expression panels, thus requiring novel maintenance strategies. Novel pharmaceutical agents may provide more frequent and longer responses such as Decitabine which is currently used in AML/MDS. Moreover, as number of other targeted therapies are at different stages of clinical trials, FLT3-inhbitors and IDH-inhibitors), monoclonal antibodies to CD33 and CD123, novel cytotoxic agents including CPX-351, Bcl2-inhibitors etc. Clinical trials of combination therapy with novel hypomethylating agents have shown an increased clinical response, when compared to single-agent demethylation treatment. In particular, combined application of FLT3-inhibitors, hypomethylating drugs and standard cytotoxic chemotherapy with cytarabine, idarubicin etc. may improve the response rate and durability of the response. Clinical trials in immune therapy are now oriented for checkpoint based combinations, AML-specific vaccines, and CART-cells to AML antigens.

Another special report by Dr. Daver was dedicated to Modern Strategies in the Treatment of Myelofibrosis including polycythemia vera (PV), primary myelofibrosis (PMF) and essential thrombocythemia (ET). Appropriate targeted treatment in myeloproliferative neoplasias is based on phenotypic driver mutations activating the JAK-STAT pathway. A number of other mutations (SRSF3, TET, AXCL1 etc.) are common in PMF. Some data from Phase 3 COMFORT-I and -II studies with Ruxolitinib in myelofibrosis are presented in the report showing some favorable effect of Ruxolitinib, with respect to overall survival and peripheral blood parameters. Appropriate combination therapy schedules are proposed for the myelofibrosis patients. Some data on Sotatercept (ACE-011), a novel soluble receptor fusion protein, are also proposed.

A session Translational investigations in hematology was mostly devoted to research and laboratory aspects of leukemias. Doctor C. Bueso-Ramos (Houston, USA) held a lecture on Laboratory diagnostics in modern oncohematology being a comprehensive presentation for practical doctors, introducing them to modern laboratory diagnostics in oncohematology. Recent improvements in laboratory testing allow to perform timely diagnostics of MDS \& AML, their providing better subtyping based, e.g., on mutation profiles, detection of novel genetic \& epigenetic drivers of neoplasia, prediction of clinical prognosis and response to therapy? Classical hematological diagnostic based on morphology 
and histochemistry is accomplished by modern molecular genetics methods. Multidimensional model for tumor classification is proposed, with regard to morphogenetic analysis, genetic progression, searching new molecular targets for etiological therapy. A workflow for bone marrow specimens and trephine biopsies is described. The 2016 Revision of WHO Classification for AML was presented, with new genetic subtypes introduced. A new type (cup-like nuclei) acute myeloid leukemia associated with FLT3 internal tandem duplication and NPM1 mutation is described. The issues of laboratory diagnostics of myelodysplastic syndromes (MDS) and their relations to AML and other disorders are discussed, along with new cytogemetic classification. Hypomethylating therapy is proven to be effective in MDS, especially, if combined with PD-1 inhibitors. A role of TP53 and other common mutations revealed by SNP panels of MDS cells is discussed. A novel approach based on plasma miRNA profiles may also help to discern distinct cytogenetic types. An integrated, "just in time”, case assessment in AML \& MDS is required, in order to discover a novel fusion genes and other oncogenic mutations.

Professor Michael Andreeff (Houston, USA) has spoken on a quite intriguing topic Leukemia cell - stromal interactions, reviewing a number of facts confirming microenvironment-induced chemoresistance of AML cells. E.g., mesenchymal stem cells (MSCs) support AML growth while changing their gene expression. Therefore, MSC signaling factors could be targeted for inhibition of leukemia growth. SDF1/CXCR4 expression correlates with decreased chemosensitivity of leukemic cells. Targeting SDF/CXCR4 is proposed for leukemic stem cells (LSCs) detachment, mobilization and apoptosis. Preclinical studies of CXCR4 inihibition or knockout were performed. Inhibition of CXCR4 or hsa-let7a miRNA overexpression lead to enhanced Ara-Cinduced apoptosis in vitro and in vivo. The patients with resistant AML and Flt mutation were treated by targeted drugs (especially Sorafenib, an Flt-3 inhibitor), in combination with Plerixafor and G-CSF. Preferential mobilization of leukemic cells was observed in this group. Silencing HIF1a may also reduce AML, as shown in murine models. In summary, stromal cells may protect leukemic stem cells from chemotherapy and TKI-induced apoptosis. Endosteal and perivascular niche are favorable for LSC growth. E.g., MSC cause resistance of leukemic cells by BCL-2 inhibition of osteoblasts. Moreover, AML cells are able to promote osteogenic differentiation, thus increasing leukemia growth support.

A sufficient discussion concerned cellular immune therapy in leukemias and lymphomas. Dr. S. Neelapu (Houston, USA) held a lecture CAR T cell therapy for lymphomas which contained some general data about technologies of generating CAR-T cells, their potential applications for treatment of different cancers, performed trials for their safety. Some adverse effects, including neurological complications, are sometimes associated with infusions of CAR T cells, and the ways of management are proposed for such situations.

Alexander V. Karabelskij and Andrey Yu. Zaritskey (St. Petersburg, Russia) have presented a report on Complex approach in CAR-T platform development and improvement. The authors reported a variety of chimeric antigen receptors (CARs) which could be applied for tumor cell recog- nition and cytotoxic treatment. In accordance to modern requirements for generation and expansion of virally-modified CART-cells, a laboratory complex for cell production is presented which is served by the Russian BioCad team, and equipped by GLP standards. The ongoing research concerns preparation of CD19-targeted CAR-T for therapy. In vitro augmentation of CAR cassette is currently attempted. Other oncotargets (HER2, MUC1 etc.) are also in scope. Searching for perspective applications for CAR-T therapy includes its combined usage with PD-1 inhibitors which showed its safety in Phase I clinical trials with solid tumors. Strategies for novel «off-the-shelf» CAR-T therapy products are developed. The ongoing problems are: rational optimization of CAR expression in T cells; standardization of the vector core bioprocess; extensive scaling of CAR-T cells expansion procedure.

\section{Acute lymphoblastic leukemia}

Dr. E. Jabbour (Houston, USA) in a lecture How I treat ALL presented novel results of current studies in ALL treatment, especially, in adult patients, in particular, combined treatment with a wide range of novel drugs. Dr. Marina Konopleva presented a novel view on high-risk group of Ph-like $B$ cell leukemias, where different JAK mutations (leading to CRLF2 overexpression), gene fusions (ABL1, ABL2, JAK2, EPOR, PDGFRB), or other gene mutations (IL7R, FLT3, RAS). Frontline therapy may be based on hyper-CVAD, augmented BFM protocols. High CRFL2 expression + JAK2 mutation is associated with adverse prognosis. The Ph-like ALLs may be treatable by appropriate kinase inhibitors (dasatinib, ruxolitinib), as well as blinatumomab and other methods of immunotherapy. Diagnostics of Ph-like B-ALL requires application of flow cytometry, cytogenetics and molecular biology methods.

Dr. Elena Parovichnikova (Moscow, Russia) has summarized in her lecture Modern ALL treatment in adults some new tendencies associated with cytogenetic and molecular stratification of the ALL patients, planning a risk-based therapy including novel antibodies and tyrosine kinase inhibitors.

Professor Alexander I. Karachunskiy (Moscow, Russia) presented his long-term studies on clinical protocols in a lecture ALL treatment optimization: Moscow-Berlin strategy, describing evolution and amendments of pediatric Moscow-Berlin protocol over last 20 years, and limits for its successful application in high-risk groups of ALL children. Role of L-asparaginase in prevention of relapses, other drugs added to the MBP may improve overall and relapse-free survival of pediatric ALL patients.

\section{Multiple myeloma}

A lecture Modern strategies in myeloma treatment presented by Professor Thierry Facon (Lille, France). Clonal evolution of combined myeloma may proceed after therapy with Melphalan and Lenalidomide; conventional treatment is, generally, followed by autologous SCT. Permanent evolution of multiple myeloma treatment is accompanied by new therapeutic targets and novel classes of drugs proposed. Along with well-known proteasome and HDAC inhibitors, some 
new monoclonal antibodies and immune checkpoint inhibitors are under trial over 2016-2017, like as some cellular vaccines and CAR-T cell systems are developed now, first of all, to treat relapsing/refractory myeloma cases. Somewhat higher OS rates were found in relapsing myeloma cases when accomplishing standard schedules with novel drugs (Carfilzomib, Ixazomib, Elotuzumumab, Daratumumab) at $>2$-year observations. Such combined (3-drug) regimens are usually more active when compared to 2 drug regimens, but several questions remain: is a triplet required for all patients; is the most active triplet the best for all patients? Moreover, the issue of cost is becoming more relevant with the current treatment regimens, due to high costs of modern therapy.

Prof. Larisa P. Mendeleeva (Moscow, Russia) reported current experience of Auto-SCT in dialysis-depended myeloma at the Federal Hematological Research Center (Moscow), especially in myeloma patients with renal failure due to myeloma cast nephropathy which may cause altered pharmacokinetics of Melphalan and other therapeutic agents. Current study was based on results of 485 auto-HSCTs in 359 patients induced with conventional protocols, with HSC harvest followed by Melphalan conditioning and auto-HSCT with subsequent maintenance with Bortezomib, or Lenalidomide. In patients resistant to these agents, Pomalidomide-based therapy cycles are effective: complete response was registered in $88 \%$ of the cases. Special problems arised with patients on hemodialysis. A possibility of canceling hemodialysis after auto-HSCT in such myeloma patients was rather low (up to $16 \%)$. Therefore, allogeneic kidney graft transplantation is a possible alternative to hemodialysis in MM patients with end-stage chronic kidney disease.

Professor Stanislav S. Bessmeltsev (St. Petersburg, Russia) presented a lecture Modern strategies in myeloma relapse treatment containing clinical data from the St.Petersburg Institute of Hematology and Transfusiology. Multiple myeloma (MM) evolves from indolent clonal disorder to active relapse which is followed by progression and/or refractory state. This course of MM requires either conventional therapy, or, in refractory cases, combined treatment with Bortezomib-, or Lenalidomide-based regimen with Dexamethasone, as a recently proposed option. Sufficiently longer OS and progression-free survival values after 2 or more relapses are shown following auto- or allogeneic HSCT combined with novel drugs Carfilzomib, Daratumumab, Ixazomib, and Elotuzumab added to standard maintenance therapy. E.g., some data from ASPIRE and ENDEAVOR studies are presented. Carfilzomib (a KRd variant) was shown to prolong progression-free survival in any tested treatment schedule. In a TOURMALINE-MM1 study, some effect of Ixazomib upon OS and PFS was registered in frames of conventional maintenance therapy, as well as Pomalidomide administration in Lenalidomide and Bortezomib-resistant patients. The favorable effect depends on the cytogenetic risk group in MM patients.

\section{Special lectures}

Professor Boris V. Afanasyev, Director, R. Gorbacheva Research Institute of Children Oncology (St. Petersburg, Russia) presented a lecture: "The place and efficacy of allo-HSCT in relapse/refractory $(r / r)$ classical Hodgkin lymphoma: anti-CD30 and PD-1 inhibitors as the bridges for improvement of outcome". The lecture was focused on the modern treatment concepts of most unfavorable variants of classical Hodgkin lymphoma based on different types of immunotherapy (monoclonal antibodies, immune check-point inhibitors, allogeneic HSCT). Firstly, in order to improve the results of therapy, it is necessary to introduce risk-adapted protocols of the $1^{\text {st }}$ line therapy (ABVD or BEACOPP) using PET/ CT scanning control. In advanced stage, new drugs significantly improve the results of therapy. Brentuximab vedotin (BV) was reported to show positive results from Phase 3 ECHELON-1 Trial in frontline advanced Hodgkin Lymphoma (HL): 2 -y OS was $82.1 \%$ vs $77.2 \%$ comparing with $\mathrm{BV}+\mathrm{AVD} v \mathrm{ABVD}(\mathrm{p}=0.035)$. An AETHERA study showed about 20\%-gain in 3-year survival for HD patients at high risk of relapse treated with BV after auto-HSCT. However, about $50 \%$ of patients exhibit resistance to $2^{\text {nd }}$ line chemotherapy. Allo-HSCT as a treatment option provides sufficient increase of PFS in HD due to "graft versus lymphoma" effect, especially in reduced-intensity conditioning regimens (RIC) (Sureda, 2008). The outcome of haplo-HSCT in Hodgkin lymphoma seems to be better than matched related SCT (Bacigalupo, 2015). PET(+) or (-) status at allo-HSCT is also of prognostic value (Moscovits, 2016).

A single center experience of R. Gorbacheva Memorial Institute of Hematology, Oncology and Transplantation in therapy of $r / r$ Hodgkin lymphoma revealed the following results: BV therapy is quite efficient in HL causing CR in 35\% of cases. Two to four BV courses are optimal for better response, however, accompanied by some adverse effects (neutropenia, neuropathy etc.). Several factors contributed to improved outcome after allo-HSCT in these patients: BV as a "bridge" for improving the disease status at the moment of allo-HSCT, RIC including Fludarabine/Bendamustine, and posttransplant Cyclophosphamide for prophylaxis GVHD. These three factors allowed increase of a 2 -year OS to $74 \%$. Current studies are also performed with PD-1 inhibitors (Pembrolizumab and Nivolumab). Overall response rate (ORR) was 69\% after Nivolumab in resistant HL patients. A phenomenon of pseudoprogression and its role in diagnostics is discussed. Current Lymphoma Response to Immunomodulatory Therapy Criteria (LYRIC) in HL is presented. Both BV and Nivolumab proved to be quite efficient in $\mathrm{HL}$ relapses after allo-HSCT. Immune therapy in R/R HL after 1st HSCT may include several options (donor lymphocyte infusions, BV, check-point inhibitors, second allo-HSCT).

Specific surgical issues of oncohematology were raised in a communication Surgical Diseases and Complications in $\mathrm{Pa}$ tients with Hematologic Malignancies by Dr. A. Artinyan, a surgeon, specialized in treatment of surgical leukemia complications. To his experience, most common surgical emergencies may evolve due to enteritis or colitis in the course of intensive chemotherapy, or resulting from severe GvHD. Some clinical examples of treating purulent conditions in immunocompromised patients were described in this report, thus arguing for a permanent dedicated surgeon at hemato-oncological clinics. 


\section{Myeloid neoplasia}

A special session on chronic myeloid neoplasias was opened by Professor Hacop Kantarjian (Houston, USA) with a lecture How I treat CML. H. Kantarjian, a leading expert of MD Anderson Cancer Center, has presented modern strategies of CML therapy evolving since 2000 to the present time by means of tyrosine kinase inhibitors (TKI) which drastically improved long-term survival in CML (from $<10 \%$ to $>90 \%$ ). Therapeutic effect in acceleration phase and blast crisis still remains low, due to drug resistance at later stages. Blast excess and basophilia, as well as clonal anomalies (iso17/17p, $3 q 26.2$ rearrangement etc.) are among adverse prognostic factors. In summary, current strategy in CML therapy is: frontline, Imatinib $400 \mathrm{mg}$ daily; Dasatinib $100 \mathrm{mg}$ daily; Nilotinib $300 \mathrm{mg}$ BID, or Bosutinib $400 \mathrm{mg}$ daily. Second/ third line includes Nilotinib, Dasatinib, Bosutinib, Ponatinib, Omacetaxine, and allogeneic SCT is considered. Other therapies may include Decitabine, Pegasys, Hydrea, Cytarabine, combined treatment with TKIs. A big IRIS study revealed similar survival with Imatinib vs Interferon+AraC in CML over 10 years. Results of ENEST study (Imatinib vs Nilotinib) are presented. In 2017, standard treatment includes Imatinib for low-risk Sokal and older pts ( $\geq 65-70 \mathrm{yrs}) ; 2^{\text {nd }}$ generation TKIs (2G-TKIs) for higher-risk Sokal until complete clinical remission, then back to Imatinib; 2G-TKIs may be used for younger pts ( $<50 \mathrm{yrs}$ ) in whom Rx DC is important. Meanwhile, benefits and drawbacks of the $1 \mathrm{G}-$ and and 2G-TKIs including long-term toxicities were discussed. From financial viewpoint, a strong justification is required to use $2^{\text {nd }}$ TKIs as frontline CML treatment (versus generic imatinib) or lower prices of second TKIs. Patients with CML should be on daily TKIs, whether in complete remission or even if $100 \% \mathrm{Ph}$-positive, in the course of CML-CP or in transformation, except for "molecular cure" conditions. In cases of AP/BP progression or TKI failure (drug resistance mutations), HSCT is strongly indicated. In summary, Imatinib, Dasatinib, Nilotinib, Bosutinib, Ponatinib, Omacetaxine present an excellent therapy for CML; clinical/hematological complete remission (CGCR) is endpoint of $\mathrm{Rx}$ improving survival; early response (3-6 mos) is predictive for favorable course; The aim terms for PCR $<10 \%$ by 6 mo, and for CGCR, by $12+$ months are only indications to change the therapy; deeper molecular responses (MMR) improve EFS; however, they do not impact transformation or survival.

A lecture Treatment-free remissions in CML by the President of European Leukemia Net (ELN), Professor Rüdiger Hehlmann (Heidelberg University, Germany) tried to answer these questions as follows: (1) Discontinuation of tyrosine kinase inhibitors (TKIs) after long-term therapy in CML can be safe if you do it right; (2) The risk is connected with BCR-ABL which causes genetic damage and promotes progression of the disorder. Previous Imatinib trial showed that 10 -year survival was $82 \%$, with $70-80 \%$ deep molecular responses $\left(\mathrm{MR}^{4}, \mathrm{MR}^{4.5}\right)$ after 10 years and more patients dying of non-CML comorbidities; thus far, high risk of relapse remained after Imatinib discontinuation. Thus life-long treatment is recommended. However, life-long TKI treatment may be accompanied by a reduction of quality of life as a consequence of mild to moderate TKI side effects. Economic consequences of life-long treatment may be also considerable. However, due to general availability of generic Imatinib, the economic burden of life-long treatment is much reduced. Moreover, TKI discontinuation is followed in about $30 \%$ by a syndrome of skeletal pain resembling polymyalgia rheumatic. The main biological issue is that $\mathrm{BCR}-\mathrm{ABL}$ persists in genomic DNA even in stable molecular remission, thus being a course of potential relapse (ISAV study). The effects of TKI discontinuation after $>3$ years of TKI therapy (EURO-SKI study) were discussed. Molecular relapse-free survival was $56 \%$ at 1 year of observation. Longer duration of Imatinib therapy (optimal $\geq 5.8$ years) and longer molecular remission prior to cessation correlates to a higher probability of relapse-free survival. Thus, in favorable cases $(20-40 \%$ of patients with low Sokal scores, chronic phase, typical BCRABL transcript) Imatinib (TKI) can be stopped safely after prolonged therapy and long-term $\mathrm{MR}^{4}$ molecular remission. However, frequent and regular standardized PCR monitoring is essential, due to sufficient risk of relapse.

Irina N. Subortseva (Moscow, Russia), Elsa Lomaia (St. Petersburg, Russia) reported their joined study Interferon-alfa in MPNs treatment concerning treatment of myeloproliferative neoplasms (MPN) by a-Interferon. This drug (rIFNa-2b) was used in MPN patients since late 80's. A number of studies showed hematological and clinical response in $80 \%$, however, causing toxic effects in $1 / 4$ of the group. Pegylated IFNs showed less toxicity, thus prompting current study of comparative efficacy and safety of Cepeginterferon alfa-2b, interferon alpha- $2 \mathrm{~b}$, and hydroxycarbamide in patients with essential thrombocytopenia and polycytemia vera $(n=63)$. JAK2 mutation was revealed in most cases. Treatment with Cepeginterferon alfa-2b caused hematological response in $78 \%$ of cases and drop in JAK2 burden, as well as improved quality of life. In summary, Cepeginterferon alfa-2b therapy in patients with MPNs is characterized by high efficacy in achieving clinical and hematological responses, and acceptable safety profile without any significant differences for these parameters with hydroxycarbamide, or $\alpha$-rINF.

\section{Stem cell transplantation}

A special session on stem cell transplantation started with a lecture by Dr. I. Khouri (Houston, USA) Modern strategies in stem cell transplantation. Dr. Khouri focused his presentation on modern treatment of non-Hodgkin's lymphomas and AML by means of allo-HSCT while mentioning age of patient, performance status and early progression as main factors predicting better clinical effect. Allo-HSCT with nonmyeloablative conditioning by Fludarabine, Rituximab, MTx (Cy) is proposed for relapsing B cell lymphoma, with longterm survival of $>80 \%$. When treating AML by allo-HSCT, minimal residual disease pre-transplant is a high risk factor for OS and RFS. Among new therapies, immunotoxic drugs (e.g., Zevalin, Tiuxetan) may be successfully used in refractory lymphomas. Rituximab may be applied together with Bendamustine and Fludarabine as pre-transplant conditioning in relapsing lymphomas. Haploidentical SCT proved to be, at least, of similar efficiency when compared to unrelated SCT, if performed with posttransplant Cyclophosphamide prophylaxis of GvHD. Brentuximab Vedotin provides better PFS rates in maintenance therapy of Hodgkin disease. 
Dr. Leslie Lehmann from Dana Farber Cancer Institute (Boston, USA), in her Transplant in pediatric leukemia patients specified three challenges in ALL treatment (decreased intensity of therapy; decreased long and short-term toxicity; perfect risk-stratification). HSCT may be effective in $1^{\text {st }}$ and further clinical remissions, dependent on absence of active disease pre-transplant. Early relapse or late relapse with $2^{\text {nd }}$ induction failure are relative indications for allo-HSCT in ALL. Primary risk factors include age of the child, leukocyte number at presentation, pre-treatment degree. Disease-dependent risk factors include ALL phenotype, cytogenetic findings, extramedullary relapse sites. Conditioning regimen should take these factors into account. Majority of survivors of pediatric HSCT are similar to age-matched controls: different from age-matched controls stature, bone health, gonadal function and reproductive health. Ongoing chronic GVHD is a biggest predictor of poor outcome. Novel therapeutic approaches, e.g., cytokine therapy, vaccine therapy, anti-PD-1 inhibitors are promising, especially, in lymphomas. CAR-T anti-CD19+ cell therapy may be applied at any stage before or after HSCT, being effective also in extramedullary lesions (e.g., brain involvement). In summary, modern view includes diminished indications for standard HSCT in childhood ALL. This procedure has to be used in terms of more precise risk stratification; targeted therapy, and better tolerated intense therapy.

Dr. Dmitry Motorin has shared clinical experience of the Almazov Medical Center (St. Petersburg) in haploidentical hematopoietic stem cell transplantation (haplo-SCT). This HSCT type is highly demanded, due to donor availability, lower costs as compared to HSCT from matched unrelated donors. Moreover, progression-free survival (PFS) in AML treatment strongly depends on duration of complete remission and molecular genetic risk group. Over last five years, a shift towards haplo-HSCT from haploidentical family donors is observed at the Almazov Center when managing acceleration phase, or blast crisis in CML, myelodysplastic syndrome/AML, and in ALL cases (male donors were preferred). Nonmyeloablative conditioning (Flu-Cy-Mel) was used in $75 \%$ of cases. GVHD prophylaxis included posttransplant Cy plus Cyclosporin A plus Mycophenolic acid. Engraftment terms, early outcomes and complications of haplo-HSCT corresponded to other alloHSCT types. OS values after allo-HSCT in CML depended on completeness of cytogenetic remission, and phase of disease at transplant. Haplo-HSCT seems to cause early decrease in activity of CML. Longer terms of observation after haplo-HSCT in AML showed a 2 -year OS of $>40 \%$. Early complications (infections, GvHD et al.) occurred at certain rates. Posttransplant Azacytidine prophylaxis in AML MRD-free patients improves progression-free survival.

Mikhail Maschan, L. Shelikhova (Moscow, Russia) presented their data on haplo-SCT in pediatric leukemia patients in Russia. Dr. Shelikhova from Dmitriy Rogachev Federal Center for Pediatric Hematology, Oncology and Immunology (Moscow) has performed allo-HSCT in 280 childhood ALL and AML patients (haplo-HSCT, in 151 cases), up to half of them performed in first remission. The grafts in 75\% of cases were subject to TCR $\alpha \beta, C D 19$ depletion which provides elimination of GVHD effectors and Epstein-Barr virus reservoire, while saving potential GVL effectors in the graft.
The 3-year EFS was 59\%, and transplant-related mortality was maintained at $8-11 \%$. Acute and chronic GvHD incidence, and general relapse rates were similar for matched unrelated and haploidentical HSCTs. Meanwhile, incidence of acute GvHD after ATGAM injections proved to be twice higher than after Thymoglobulin usage. A 3-year event-free survival in AML (from complete remission state) was higher after haplo-HSCT (86\%) than following unrelated matched HSCT (55\%). Hence, in both AML and ALL settings, haplo-HSCT should be preferred to MUD.

In a report by Tatyana Bykova, Alexander D. Kulagin (St. Petersburg, Russia) Allo-HSCT for aplastic anemia (AA) and paroxysmal nocturnal hemoglobinuria $(P N H) / A A$. Dr. T. Bykova reported current data from Raisa Gorbacheva Memorial Institute of Children Oncology, Hematology and Transplantation concerning allo-HSCT in aplastic anemia (AA), AA/ $\mathrm{PNH} /$, and AA/MDS/AML (a total of 90 cases) including 8 haplo-HSCT. Most common conditioning was FLU/CY(Bu)/ ATG or ALEM. Secondary graft failure at 2 years was recorded in $18 \%$ of transplanted patients. Severe acute GvHD occurred in 5\% of cases, whereas chronic GvHD was more common upon grafting with peripheral blood stem cells. Overall survival was much higher after HSCT from matched siblings. Eculizumab as anti-hemolytic treatment was used in 16 cases as bridging therapy in AA/PNH before HSCT. Engraftment was reached at a mean of 20 days correlating with decrease in $\mathrm{PNH}$ clone extent. Secondary graft failure was revealed in $9 \%$ of cases. Severe GvHD was no detected, disease-free survival was $84 \%$ at 2 years. In conclusion, allogeneic BMT proved to be a standard curative option for severe AA; bone marrow is a preferred source of stem cells. Eculizumab bridging for HSCT is preferrable for hemolytic PNH/SAA therapy.

Ivan S. Moiseev (St. Petersburg, Russia) told about Novel approaches in the prophylaxis and treatment of GVHD after Allo- HSCT. He has followed evolution of aGVHD prophylaxis $\mathrm{n}$ allo-HSCT, from Cyclosporin A+Methotrexate to Tacrolimus and MMF implementation. Haploidentical HSCT proved to be a favorable option to avoid severe aGvHD. In this respect, a trial of aGvHD prophylaxis with posttransplant cyclophosphamide (PTCy) was started at the R. Gorbacheva Research Institute of Children Oncology (trial No.NCT02294552). When comparing injections of horse ATG with PTCy prevention, the latter regimen was associated with lower incidence of acute and chronic GvHD, thus transplating in lower survival probability. Early event-free survival proved to be higher with single-agent PTCy than in $\mathrm{CNI} / \mathrm{MMF} / \mathrm{MTx}$ regimen. An NCT02627573 trial started in 2015 , been designed as a randomized trial of GVHD prophylaxis with PTCy vs. Thymoglobulin in unrelated SCT with chronic myeloproliferative neoplasms and myelodysplatic syndrome. Extracorporeal cytopheresis and anticytokine therapy were compared for chronic GvHD therapy, the study is in progress.

\section{Conflict of interest}

No conflict of interests is declared. 


\section{Отчет о международной конференции «Современные стратегии лечения и горизонты гематологии и онкологии» (30 июня - 1 июля 2017 г.)}

\section{Андрей Ю. Зарицкий ${ }^{1}$, Борис В. Афанасьев ${ }^{2}$, Дмитрий В. Моторин ${ }^{1}$}

${ }^{1}$ Национальный медицинский исследовательский центр имени В. А. Алмазова, Санкт-Петербург, Россия

${ }^{2}$ Научно-исследовательский институт детской онкологии, гематологии и трансплантологии им. Р. Горбачевой, Первый Санкт-Петербургский государственный медицинский университет им. И. П. Павлова, Санкт-Петербург, Россия

\section{Резюме}

Совместная конференция клиницистов медицинского Центра M.D. Anderson (Хьюстон, Техас, США) и специалистов ведущих российских гематологических центров состоялась в Санкт-Петербурге 30 июня - 1 июля 2017 г. Ряд отдельных заседаний был посвящен текущим проблемам диагностики и патогенетической молекулярной классификации в гематоонкологии, в том числе - при хроническом лимфолейкозе, острых лейкозах, апластических анемиях (АА), миелодиспластическом синдроме (МДС), злокачественных лимфомах. Протоколы стандартного лечения, а также клиническая эффективность новых таргетных препаратов и комбинированные терапевтические подходы подвергались рассмотрению с акцентом на лечение болезни Ходжкина, хронического лимфолейкоза, хронического миелоидного лейкоза и миеломной болезни.
Были также представлены современные разработки в лекарственном лечении острых лейкозов, особенно - острого миелобластного лейкоза. Проводилось рассмотрение и обсуждение эффективности трансплантации гемопоэтических стволовых клеток в качестве излечивающей терапии при некоторых формах лейкозов и лимфом, а также возможности иммунотерапии опухолей (например, CAR-T клетками) и соответствующие технологии.

\section{Ключевые слова}

Российско-американская конференция, лейкозы, лимфомы, патогенез, классификация, лечение, таргетные препараты, аллогенная трансплантация гемопоэтических стволовых клеток. 